\title{
Integrated luminescent chemical microsensors based on GaN LEDs for security applications using smartphones
}

\author{
Guillermo Orellana Elías Muñoz Luz K. Gil-Herrera Pablo Muñoz Juan López-Gejo Carlos \\ Palacio
}

\begin{abstract}
Development of PCB-integrateable microsensors for monitoring chemical species is a goal in areas such as lab-on-a-chip analytical devices, diagnostics medicine and electronics for hand-held instruments where the device size is a major issue. Cellular phones have pervaded the world inhabitants and their usefulness has dramatically increased with the introduction of smartphones due to a combination of amazing processing power in a confined space, geolocalization and manifold telecommunication features. Therefore, a number of physical and chemical sensors that add value to the terminal for health monitoring, personal safety (at home, at work) and, eventually, national security have started to be developed, capitalizing also on the huge number of circulating cell phones. The chemical sensor-enabled "super" smartphone provides a unique (bio)sensing platform for monitoring airborne or waterborne hazardous chemicals or microorganisms for both single user and crowdsourcing security applications. Some of the latest ones are illustrated by a few examples. Moreover, we have recently achieved for the first time (covalent) functionalization of $p$ - and $n-G a N$ semiconductor surfaces with tuneable luminescent indicator dyes of the Ru-polypyridyl family, as a key step in the development of innovative microsensors for smartphone applications. Chemical "sensoring" of GaN-based blue LED chips with those indicators has also been achieved by plasma treatment of their surface, and the micrometer-sized devices have been tested to monitor $\mathrm{O}_{2}$ in the gas phase to show their full functionality. Novel strategies to enhance the sensor sensitivity such as changing the length and nature of the siloxane buffer layer are discussed in this paper.
\end{abstract}

Keywords: Chemical sensors, luminescence, gas detection, GaN LEDs, smartphone, advanced materials, surface functionalization, ruthenium complexes

\section{INTRODUCTION}

Development of PCB-integrateable microsensors for monitoring chemical species is a top goal in scientific and technical areas such as lab-on-a-chip analytical devices, diagnostics medicine and electronics for hand-held instruments where the device size is a major issue. Cellular phones have pervaded the world inhabitants becoming an extension of ourselves without which we cannot leave home. In many parts of the world more people have access to a mobile device than to a toilet or sanitized water. Their usefulness has dramatically increased with the introduction of smartphones due to a combination of amazing processing power in an extremely confined space ("pocket-sized"), geolocalization and manifold telecommunication features. A typical current smartphone carries more computing power than the Apollo 11 rocket that landed men on the moon. Best of all, prices of smartphones are dropping an average of $41 \%$ after just four months of a phone's launch date in the US. ${ }^{1}$ Therefore, it is no surprise that major manufacturers and service providers rest their glaze on physical and chemical sensors that may add value to their devices and offerings for easing the personal daily life. At the same time, the huge number of circulating cell phones provides a unique (bio)sensing network for monitoring airborne or waterborne hazardous chemicals or microorganisms over very wide areas for personal (home) and industrial workers safety as well as national security applications (by crowdsourcing). The world's 4 billion mobile terminals could even be turned into sensors on a global data-collection network!. No doubt micro- and nanotechnology are expected to play a pivotal role in the miniaturization required for printed circuit board (PCB)-integrateable sensors inside phones, or attached to them. ${ }^{2}$ As chips become smaller and more advanced, cell phones will take in new functions.

*orellana@quim.ucm.es; phone +34 913944 220; fax +34 913944 103; www.ucm.es/info/gsolfa 
A quick look at the current situation already provides hints of what we are heading for. Leaving aside already cell phoneembedded physical sensors such as position, tilt or motion, health-oriented applications have been the first to take the lead, mostly (so far) with the user intervention. ${ }^{3}$ For instance, non-profit Innovative Support to Emergencies, Diseases and Disasters (InSTEDD)'s GeoChat (instedd.org) is a collaboration tool designed to enable self-organizing group communications by allowing users to link the field, headquarters and the local community in a real-time, interactive conversation visualized on the surface of a map. It is based on SMS, email and Twitter over any device, platform or network. Among many communities worldwide, it has been officially adopted by the six countries which share a border in the Mekong Basin, establishing in this way a flow of real-time disease data from villages in the region to each country's health ministry. Authorities can then choose to share this information with international bodies such as the US Centers for Disease Control (CDC) or the World Health Organization. The aim is to provide a quick response to any outbreak of avian flu, cholera, malaria or dengue fever. Sense Networks (sensenetworks.com) MacroSens ${ }^{\mathbb{B}}$ technology platform, however, unattendedly extracts behavioral information from location and applies predictive analytics without personal information. It may be used to send personalized adds but also for medical and security applications.

More focused on true (bio)chemical sensing for e-health uses, the Lifelens project (lifelensproject.com/blog/) has created an innovative point-of-care smartphone application that addresses child mortality rates caused by the lack of detection and availability of treatment for malaria. The current state-of-the-art rapid diagnostic tests deployed in many less-favored regions of the world have ca. a $60 \%$ rate of false positives yielding unnecessary treatments and increasing the likelihood of drug resistance. The vision of Lifelens project is to digitally characterize anemia, visualize blood cell rupture and parasites, and provide three-dimensional modeling of cells through single image acquisition of low-volume blood smears by peripheral finger pricks. The Lifelens' Smartphone app aims to be more than $95 \%$ accurate in the tests run by anyone who has the ability to operate a basic smartphone. Lifelens devices are equipped with proprietary image analysis algorithm written in .NET with Visual Studio for Windows 7 phones which can detect the presence of a malaria parasite within the patient's blood cells through a tiny lens giving $350 \mathrm{X}$ magnification. The drop of blood is placed on a slide with a marker dye that only the target parasite can absorb, for cell counting over the image captured in the smartphone. ${ }^{4}$

The Nokia Research Center recently initiated a project for a futuristic mobile phone concept ("Nokia Eco Sensor") with an aim to initiate public discussion around environmentally sustainable monitoring with broad public involvement (research.nokia.com/news/564). It might include a separate, wearable sensing device with detectors that collect environment, health, and/or weather data. Information is transferred to the smartphone using Near Field Communications technology. Unfortunately, no further details have been made available on the technologies used for chemical sensing.

Originated by the US Department of Homeland Security's Science and Technology Directorate (S\&T), the Cell-All initiative aims to equip a cell phone with a sensor capable of detecting deadly chemicals at minimal cost - to the manufacturer (a buck a sensor) and to the smartphone's battery life. ${ }^{5}$ The sensing phone will regularly sniff the surrounding air for certain volatile chemical compounds. When a personal threat is detected, such as a chlorine gas leak, a warning is sounded; the user can choose a vibration, noise, text message, or phone call. For situations that may compromise national security, such as a sarin gas attack, details - including time, location, and the compound - are phoned home to an emergency operations center. This second type of warning works best with many phone bearers concentrated in a particular area so as to avoid false positives and to mark out boundaries of the chemical threat (unattended crowdsourcing human safety). S\&T is actively funding R\&D in this area to see if the concept is workable thanks to their respective area of expertise of three teams from Qualcomm (miniaturization), the National Aeronautics and Space Administration (NASA, Ames Research Center; chemical sensing on low-powered platforms), and Rhevision Technology (artificial optical noses). Cooperative research and development agreements with cell phone manufacturers such as Qualcomm, LG, Apple and Samsung is expected to accelerate commercialization of the developed technology.

In the meantime, several patents have been filed unveiling technologies that might boost realization of the cell-phone-asa-sensor concept. For instance, Wang and Zhou (BWT Property Inc., NJ) ${ }^{6}$ disclose a spectroscopic sensor that is integrated with any mobile communication device. The sensor is capable of measuring the optical spectrum of a physical object for purposes of detection, identification, authentication and real time monitoring. Such a sensing element may comprise a photodiode, CCD or CMOS detector, a wavelength-selective element and a LED or laser diode light source, all integrated in a camera mobile phone. Reflection, scattering or fluorescence optical signals may be used to the purpose. Practical examples of applications of such a device include real-time monitoring of blood oxygenation levels by the VIS-NIR absorption spectrum of living human tissue (measuring the ratio of the oxyhemoglobin to hemoglobin content at characteristic wavelengths), the level of protein, lipid, carotenoid or other chemicals in the skin by the Raman 
scattering spectrum, the variations in the sunlight spectrum after atmospheric transmission, and detection of hazardous materials at airports, storehouses or the battlefield.

A recent Chinese patent ${ }^{7}$ discloses a mobile phone with an alcohol detection function for personal safety. The device comprises a smartphone body, an alcohol gas sensor, an analog-to-digital converter and a baseband chip. Moreover, a blow port is set at the top of the mobile phone body to allow the user check the alcohol contents of his/her breathe. For industrial workers' personal safety, the Korean company SF Technology has filed a patent describing a smartphoneintegrated environmental radioactivity measuring device. ${ }^{8}$ The latter comprises a web application installed for radioactivity measurements, a USB transmitting/receiving port and a data transmission module. The radioactivity sensor is connected to the USB port and is able to measure in real time.

Chemical sensors and biosensors for smartphone applications are also starting to appear in the scientific/technical literature. In most cases, however, the authors have used components of the same type that those typically found in mobile devices or compatible with them rather than building an operational terminal. For instance, B.-Y. Chang describes smartphone-based chemistry instrumentation for digitizing colorimetric measurements employing its built-in functions (embedded CCD camera, software, transmission, ...). ${ }^{9}$ The "mobile analytical transducer" concept has been demonstrated with a Samsung Galaxy and $\mathrm{pH}$ (litmus) paper, the color change of which is measured with a snapshot rather than the bare eye for increased accuracy and precision.

López-Ruiz et al. have manufactured a portable instrument for $\mathrm{O}_{2}$ measurements in the gas phase based on the platinum octaethylporphyrin luminescence (intensity) quenching by the analyte. ${ }^{10}$ The indicator dye is excited with a $380 \mathrm{~nm} \mathrm{UV}$ LED and the emission from a polymer film containing the indicator dye is recorded by a CCD micro-camera. This 2-D image is processed by a microcontroller to obtain the red colour component of the RGB colour space which is related to the concentration of the surrounding $\mathrm{O}_{2}$. With the future aim of using the developed technology in smartphones, an application for camera-enabled Android devices was also developed.

A similar approach for biosensing has been tested by Marimuthu et al. ${ }^{11}$ They have evaluated the innovative way of using a CMOS image sensor as an immunosensing device for detecting the interaction between recombinant interferon gamma (IFN- $\gamma$ ) antigen and selective primary and secondary IFN- $\gamma$ antibodies adsorbed onto In nanoparticles-coated glass substrates. By counting the difference in photon number using the CMOS image sensor after antigen and antibody binding, they tried to measure the potential use of this technique in smartphones or clinical diagnostic devices for portable personal health care systems. The metal nanoparticles facilitated light scattering effects, which are essential in the detection of antigen-antibody interactions at concentrations down to $1 \mathrm{fg} / \mathrm{mL}$ by the image sensors.

Semiconductor-based light-emitting devices (LEDs) are nowadays present in everyday's life including domestic appliances, industrial instruments and illumination because of their long operational lifetime, output intensity and low cost. Their current emission wavelengths span from the UV to the near-infrared, but blue-violet LEDs are arguably the most attractive sources for fluorescence excitation of indicator dyes due to the absorption bands that most of them show in this region. GaN-based semiconductors are the cornerstone of blue LEDs because powerful chips have been developed (including laser diodes) and their emission wavelength can be tuned in a wide range if $\mathrm{Al}$, In, or other metals are introduced in the GaN lattice. ${ }^{12}$

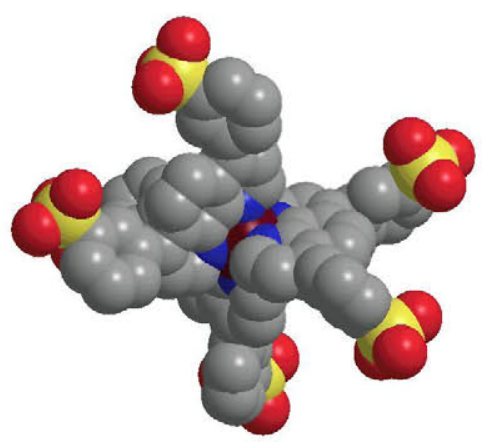

Figure 1. Chemical structure (CPK model depicted) of the luminescent indicator dye tetrasodium tris[[(1,10-phenanthroline4,7-diyl- $\kappa N^{1}, \kappa N^{10}$ ) bis(benzenesulfonato) $\left.](2-)\right]$ ruthenate(4-), abbreviated $\mathrm{Na}_{4}\left[\mathrm{Ru}(\mathrm{pbbs})_{3}\right]$. The sodium counterions have been omitted because they do not contribute to its emission features. The precursor commercial ligand is actually a mixture of structural isomers with the sulfonate groups in the 3' and 4' positions. No attempt to separate such a mixture was made. 
Recently, ${ }^{13}$ we achieved for the first time (covalent) functionalization of semiconductor $p$ - and n-GaN surfaces with tuneable luminescent indicator dyes of the Ru-polypyridyl family (Figure 1) ${ }^{14}$ as a key step in the development of innovative microsensors for smartphone applications. Coordination complexes of $\mathrm{Ru}(\mathrm{II})$ with polyazaheteroaromatic chelating ligands are very suitable luminescent indicator dyes for chemical sensing due to an almost unique combination of well-separated excitation and emission wavelengths (blue/red), significant emission quantum yields (up to 0.5), long excited state lifetimes (up to ca. $7 \mu \mathrm{s}$ in solution at room temperature in the absence of any quencher), and extraordinary photochemical/thermal stability compared to purely organic dyes. Moreover, their sophisticated structure allows tweaking their photochemistry to make them sensitive to vastly different analytes by either photoinduced energy-, electron- or proton-transfer processes. Modification of the coordinating ligands also allows changing dramatically their overall electrical charge, solubility, hydrophobicity and polymer supports for an optimal immobilization and fabrication of the chemically-sensitive element. ${ }^{14,15}$

In the course of our research, we found that only functionalized p-GaN was useful for photochemical monitoring of chemical species due to the (unexpected) photoinduced electron transfer quenching of the surface-tethered luminescent indicator dye on n-GaN. Chemical "sensoring" of InGaN/GaN blue LED chips with those metal-organic indicators has also been achieved by plasma treatment of their surface, and the resulting micrometer-sized prototypes were tested as a proof-of-concept to monitor $\mathrm{O}_{2}$ in the gas phase, showing their full functionality. ${ }^{16}$ Although InGaN/GaN LED have usually a p-GaN uppermost layer, recent high-efficiency chip designs have moved to a top n-GaN layer. ${ }^{17}$ Aiming to explore for general sensor-enabled semiconductor light sources, we have sought novel strategies to eliminate or decrease the rate of such photochemical competitive deactivation pathway in order to increase the device sensitivity. Preliminary results on the effect of the thickness and nature of the siloxane buffer are presented in this paper.

\section{EXPERIMENTAL SECTION}

Ca. $8 \times 8 \mathrm{~mm}$ samples of n-type GaN were cut from a commercial substrate (wafers from Lumilog, France). Preparation of the $\mathrm{Ru}(\mathrm{II})$ luminescent indicator dye $\mathrm{Na}_{4}\left[\mathrm{Ru}(\mathrm{pbbs})_{3}\right]$, cleaning and oxygen plasma activation of the $\mathrm{n}$-GaN surface, silanization of the semiconductor with $\gamma$-aminopropyltriethoxysilane (APTES, Sigma-Aldrich), and the procedure for covalent attachment of the dye to the layer of siloxane molecules by making a strong sulfonamide bond, have been described. ${ }^{13,16}$ APTES was replaced by $\gamma$-aminopropyltrimethoxysilane (APTMS), 4,7-diazaheptyltrimethoxysilane (DAHTMS) or 4,7,10-triazadecyltrimethoxysilane (TADTMS), respectively, to prepare dye-functionalized $n$-GaN samples with different siloxane linkers (all from $\mathrm{ABCR}$, Germany).

Luminescence lifetime measurements of the functionalized n-GaN samples were performed on a single-photon timing (SPT) fluorometer (Horiba Fluoromax-4SPT, NJ, USA) using laser diode excitation at $463 \mathrm{~nm}$ (Horiba NanoLED 470LH source, Glasgow, UK). Variable delay times with respect to the trigger pulse could be adjusted with an external gate and delay generator (Ortec 416A, TN, USA). Luminescence decays $I(t)$ were measured with a $10 \mu$ s or $20 \mu$ sindow by accumulating the signal over at least 5000 counts in the peak channel. Pre-exponential weighted mean emission lifetimes $\left(\tau_{m}=\sum_{i}\left(\%_{i} \tau_{i} / 100\right)\right)$ were obtained thereof after tri-exponential $(i=3)$ curve fittings $\left(I(t)=A+\sum_{i} B_{i} e^{-t / \tau_{i}}\right)$ using the proprietary Horiba hybrid grid-search minimization algorithm (without deconvolution) for stable chi-squared minimization. The reduced $\chi_{\mathrm{r}}^{2}(<1.1)$ and weighted residuals functions were employed to judge the goodness-of-the-fits. Measurements were carried out at $(711 \pm 5)$ Torr of argon gas from cylinders (Praxair, Madrid, Spain).

\section{RESULTS AND DISCUSSION}

GaN can be successfully functionalized for chemical microsensing applications by covalent attachment of luminescent indicator dyes using oxygen plasma to introduce hydroxyl groups onto the semiconductor surface. ${ }^{16}$ Angle-resolved XPS experiments have demonstrated that a dense siloxane film, several monolayers thick, is formed on the n-GaN surface after hydroxylation and silanization. ${ }^{18}$ Evidence of formation of a sulfonamide bond between the metal-organic indicator dye and the silanized semiconductor surface is extracted from the analysis of the $S 2 p$ core level $X$-ray photoelectron 
spectra (XPS) because it is slightly different in the sulfonamide and in the original sulfonate groups. ${ }^{13,16}$ Occurrence of a reversible photooxidation of the surface-bound $\mathrm{Ru}$ (II) luminescent dye has been suggested from the disparate values of its emission lifetime on p-GaN (ca. $2 \mu$ s under argon) and on n-GaN (ca. $0.6 \mu$ s under argon). ${ }^{16}$ Although the exact origin of the excited state quenching mechanism has not been unequivocally elucidated yet (e.g. by detecting the transient $\mathrm{Ru}$ (III) species using laser kinetic spectrometry), deactivation of the photoexcited dye through Förster resonance energy transfer (FRET) has to be ruled out due to the absence of spectral overlap between the Ru(II) complex (donor) and the GaN (acceptor). Nevertheless, energy transfer by a Dexter-type (electron exchange) mechanism might occur if the ionization potentials (IPs) and electron affinities (EAs) of the photoexcited dye and the semiconductor are adequate.

To further investigate this issue, a literature search for the IPs and EAs of a number of relevant n-type semiconductor materials (plus $\mathrm{SiO}_{2}$ ) has been carried out (Table 1), and the corresponding bandgaps have been all depicted in Figure 2 for an easier visualization. Figure 2 also depicts the IP and EA of $\mathrm{Na}_{4}\left[\mathrm{Ru}(\mathrm{pbbs})_{3}\right]$ calculated from the ground state oxidation potential of the complex $\left(\mathrm{D}^{+} / \mathrm{D}=1.41 \mathrm{~V}\right.$ vs NHE$),{ }^{19}$ its emission maximum $(618 \mathrm{~nm})$ and the equivalence: $0 \mathrm{~V}$ vs NHE $=4.5 \mathrm{eV}$ vs. vacuum. ${ }^{20}$

Table 1. Electron affinity, ionization potential and bandgap (eV, in vacuo) of different (n-type where applicable) materials relevant to this study.

\begin{tabular}{lllll}
\hline Material & $\begin{array}{l}\text { Bandgap } \\
(\mathbf{e V})\end{array}$ & $\begin{array}{l}\text { Electron } \\
\text { affinity }(\mathbf{e V})\end{array}$ & $\begin{array}{l}\text { Ionization } \\
\text { potential }(\mathbf{e V})\end{array}$ & Reference \\
\hline $\mathrm{GaN}$ & 3.5 & 4.1 & 7.6 & www.ioffe.ru/SVA/NSM/Semicond/ \\
$\mathrm{In}_{0.18} \mathrm{Ga}_{0.82} \mathrm{~N}$ & 2.55 & 3.73 & 6.28 & {$[21]$} \\
$\mathrm{In}_{0.26} \mathrm{Ga}_{0.74} \mathrm{~N}$ & 2.1 & 4.03 & 6.13 & {$[21]$} \\
$\mathrm{AlN}$ & 6.03 & 0.6 & 6.08 & www.ioffe.ru/SVA/NSM/Semicond/ $\quad$ (wurtzite) \\
$\mathrm{Al}_{0.3} \mathrm{Ga}_{0.7} \mathrm{~N}$ & 4.2 & 1.9 & 6.1 & {$[22]$} \\
$\mathrm{ZnO}$ & 3.4 & 4.2 & 7.6 & {$[23]$} \\
$\mathrm{TiO}_{2}$ & 3.2 & 4.5 & 7.7 & {$[24]$} \\
$\mathrm{SiO}$ & 9 & 1 & 10 & inmmc.org/ftp/material/silicon-dioxide.html \\
$\mathrm{ITO}$ & 3.6 & 4.1 & 7.7 & {$[25]$} \\
$\mathrm{InN}$ & 0.65 & 4.7 & 5.35 & {$[26]$} \\
\hline
\end{tabular}

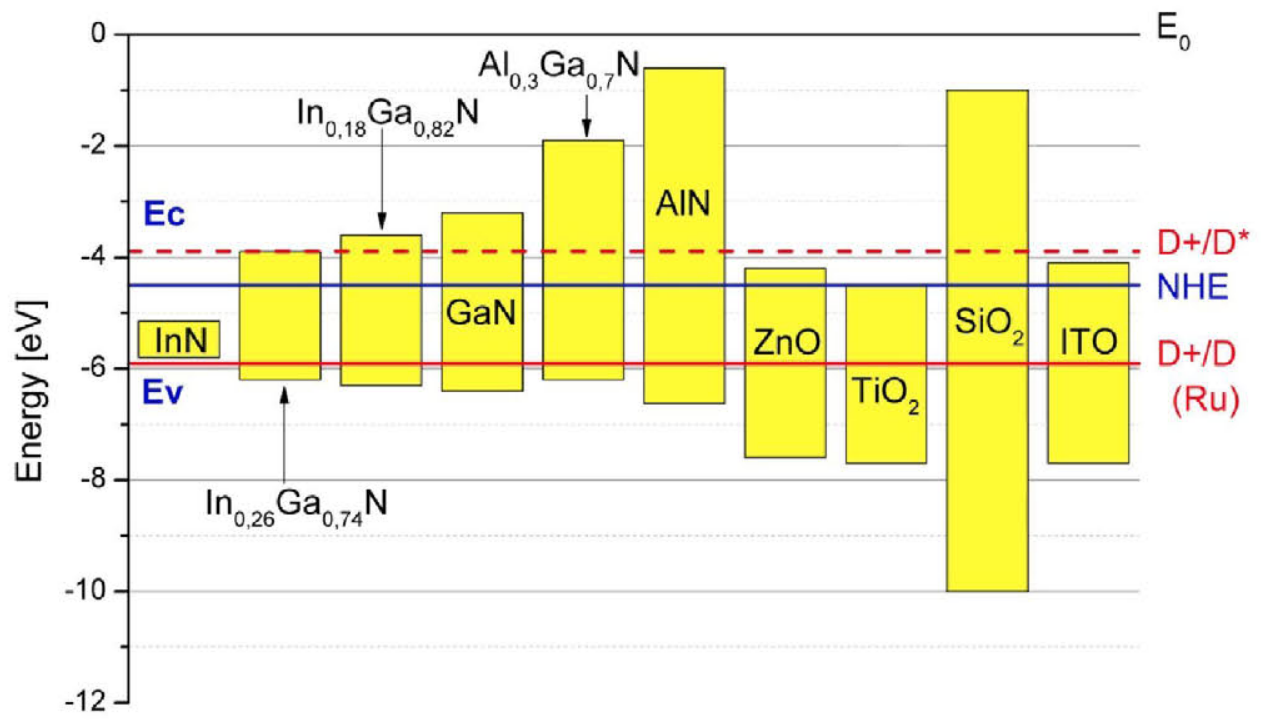

Figure 2. Band position and bandgap (in eV) of different materials relevant to this study. The diagram also depicts the ground and excited state oxidation potentials (in $\mathrm{eV}$ ) of $\mathrm{Na}_{4}\left[\mathrm{Ru}(\mathrm{pbbs})_{3}\right]$ and the redox potential of the Normal Hydrogen Electrode (NHE). All values have been taken from literature or calculated by an appropriate correction to be expressed in vacuo (see text and Table 1). 
Although the position of the conduction band of $\mathrm{n}-\mathrm{GaN}$ seems to be slightly above the oxidation potential of the Ru(II) indicator dye, precluding a PET process from it, the situation might be reversed when considering the unavoidable uncertainty due to the conversion of redox potentials in $\mathrm{V}$ (measured in aqueous solution) into $\mathrm{eV}$ (in vacuo) by assuming that the work term associated with the transfer of solvated ions into the gas phase is the same for the normal hydrogen electrode (NHE) and for the $\left[\mathrm{Ru}(\mathrm{pbbs})_{3}\right]^{4}$ species. Introducing some $\mathrm{Al}$ into the semiconductor $\left(\mathrm{Al}_{0.3} \mathrm{Ga}_{0.7} \mathrm{~N}\right)$ would be expected to suppress or decrease the PET contribution to the deactivation of the indicator excited state (Figure 2 ); conversely, allowing $\mathrm{GaN}$ with Indium $\left(\mathrm{In}_{0.18} \mathrm{Ga}_{0.82} \mathrm{~N}, \mathrm{In}_{0.26} \mathrm{Ga}_{0.74} \mathrm{~N}\right)$ should accelerate the PET process. We are currently exploring such n-type materials to shed some light into this exciting novel photochemistry.

After the GaN functionalization process, a kind of heterostructure is formed, requiring the alignment of the Fermi level $\left(E_{\mathrm{F}}\right)$ along the various layers under no external excitation. Under this condition, typically, for n-type $\mathrm{GaN}$ the conduction band (CB) is about $150 \mathrm{meV}$ above $E_{\mathrm{F}}$, while for p-type $\mathrm{GaN}, E_{\mathrm{F}}$ is about $250 \mathrm{meV}$ about the valence band. This implies that for $\mathrm{p}-\mathrm{GaN}$ the conduction band energy has risen considerably $(>3 \mathrm{eV})$ in comparison to the $\mathrm{CB}$ in n-type GaN, making very unfavourable the PET process by the photo-excited electrons in the Ru-based dye. This is the model we are considering to explain our results.

Regardless the exact mechanism that accounts for the striking difference between the behavior of *[Ru(pbbs) $] @$ @ $-\mathrm{GaN}$ vs*[Ru(pbbs) ${ }_{3} @$ a p-GaN and its effect on chemical sensing of target analytes, increasing enough the thickness of the siloxane layer sandwiched between the semiconductor surface and the luminescent indicator molecules would be expected to lead to suppression of the photoredox reaction. To investigate this intriguing possibility, we have prepared three novel $\mathrm{n}-\mathrm{GaN}$ semiconductors covalently functionalized with the $\left[\mathrm{Ru}(\mathrm{pbbs})_{3}\right]$ dye (namely DAHTMS, TADTMS and APTMS). Compared to the $\gamma$-aminopropyltriethoxysilane, the diazaheptyl and triazadecyl $\omega$-aminoalkyl siloxanes bear a ca. two-fold and three-fold longer spacer chain, respectively, between their points of attachment to the Ru complex and the hydroxylated GaN surface. $\gamma$-Aminopropyltrimethoxysilane was also used as a control experiment to check the effect of the chemical reactivity of the precursor siloxane moiety on the fabricated silane layer.

Figure 3 shows the SPT luminescence decay measurements of the new $\mathrm{n}-\mathrm{GaN}$ materials for $\mathrm{O}_{2}$ sensing under molecular oxygen-free conditions. The emission lifetime of the sample manufactured with APTES $(0.6 \mu \mathrm{s})$ is in agreement with that reported previously. ${ }^{16}$ According to our predictions, the sample fabricated with the two-fold longer silane DAHTMS displays an excited state lifetime much longer $(1.3 \mu \mathrm{s})$ that the APTES one. This result is compatible with a significant reduction in the PET rate due to the larger distance between the donor and the acceptor species (Figure 4). For the sake of comparison, the luminescence lifetime of the $*\left[\mathrm{Ru}(\mathrm{pbbs})_{3}\right]$ dye onto a glass surface, where a PET process is completely forbidden, is ca. $2.0 \mu \mathrm{s}$. Surprisingly enough, the 3 -fold longer spacer used in the TADTMS sample leads to a dramatic decrease of the $*\left[\mathrm{Ru}(\mathrm{pbbs})_{3}\right]$ emission lifetime $(0.24 \mu \mathrm{s})$ pointing out to an accelerated PET process, while the $\mathrm{n}$-GaN sample manufactured with APTMS displays a 50\% longer luminescence lifetime than its APTES analogue.

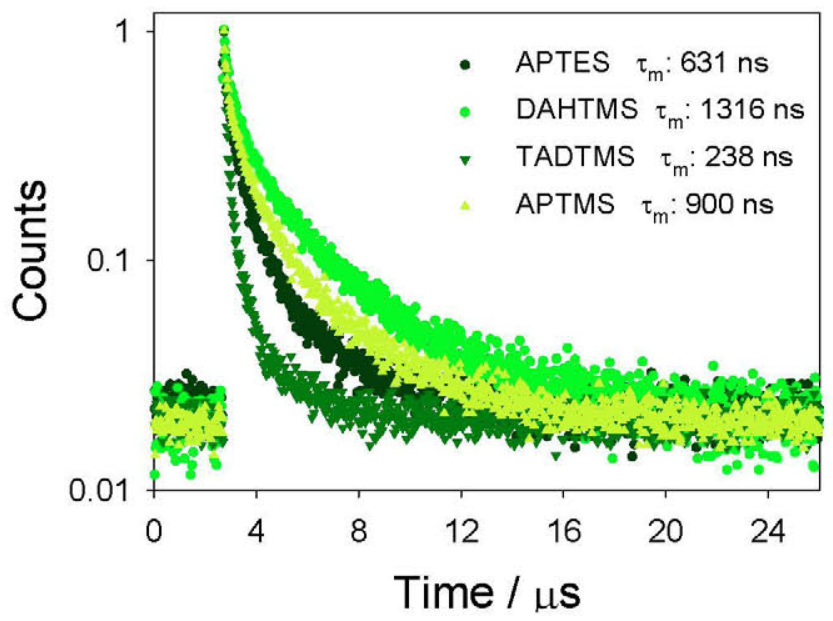

Figure 3. Kinetics of the excited state deactivation of the $\mathrm{Ru}$ (II) indicator dye covalently attached to n-GaN as a function of the $\omega$-aminoalkylsiloxane buffer layer. All decays have been recorded in an $\mathrm{O}_{2}$-free atmosphere under argon. The abbreviations of the corresponding siloxanes are given in the Experimental Section. 
An explanation of the much shorter excited state lifetime of $\left[\mathrm{Ru}(\mathrm{pbbs})_{3}\right] @ \mathrm{a}-\mathrm{GaN}$ containing TADTMS is depicted in Figure 4: the two secondary amino groups (probably protonated as a result of the functionalization reaction) interact strongly with the hydroxylated GaN surface leading to a sharp bending of the alkyl spacer chain, actually approaching and not separating the donor metal complex and the semiconductor acceptor. Under these conditions, the PET reaction rate increases with respect to the APTES-based material. How can substitution of methoxide for ethoxide groups in the silane molecule lead to a significant decrease of the PET rate? The much higher reactivity of APTMS towards substitution reactions at the Si atom compared to the ethoxysilane due to the smaller steric effect of the methoxy group would lead to formation of a thicker and/or denser alkylsiloxane layer between the $\mathrm{Ru}$ dye and the semiconductor surface, thereby decreasing the PET rate between them.

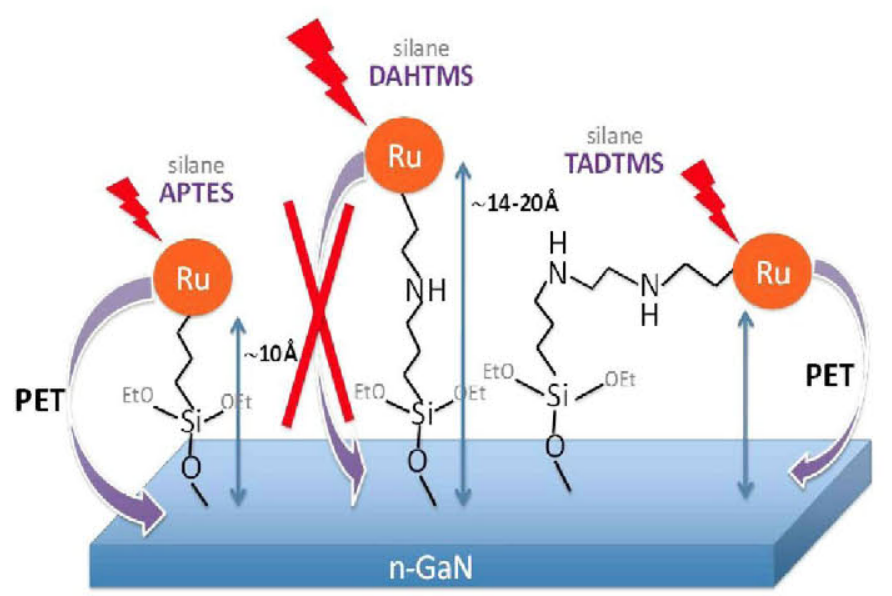

Figure 4. Proposed mechanism for inhibition or significant reduction of the photoinduced electron transfer (PET) rate from the indicator dye to the semiconductor surface by varying the siloxane buffer layer used to chemically attach the former.

\section{CONCLUSIONS}

Functionalization of blue-emitting GaN substrates with tailored luminescent Ru(II) polypyridyl complexes by way of hydroxylation, $\omega$-aminoalkylsilanization of the resulting surface, and sulfonamide derivatization with the indicator dye is a useful way of manufacturing (ultra)micro devices for optical chemical sensing. An interfering photoinduced electron transfer reaction from the tethered luminescent dye to the semiconductor has been found for n-type GaN substrates. An adequate selection of the siloxane interlayer precursor and/or the semiconductor doping itself may decrease or completely eliminate the undesired photochemistry leading to chemical sensors with enhanced sensitivity towards the target analyte. Further research is currently underway in our laboratories to develop newer Ru@GaN-based optosensors for chemical analysis with smartphone embedding.

\section{ACKNOWLEDGEMENTS}

This work is currently supported by the Spanish MICINN (CTQ2009-14565-C03-01) but has received previous funding from the Madrid Community Government (S-0505/AMB-0374) and UCM-Santander (GR35/10-A). It belongs to the "Moncloa Campus of Excellence" initiative, a joint partnership of Complutense University of Madrid and Polytechnical University of Madrid which share a common campus and research goals in the areas of Materials Research, Global Climate Change and Environmental Monitoring, among selected others. 


\section{REFERENCES}

[1] Tofel, K. C., "As prices drop, when's the best time to buy a new phone?," Gigaom, 14 May 2012, < http://gigaom.com/mobile/as-prices-drop-whens-the-best-time-to-buy-a-new-phone/> (29 August 2012).

[2] Ryhänen, T., Uusitalo, M. A., Ikkala, O. and Kärkkäinen, A., Eds., [Nanotechnologies for Future Mobile Devices], Cambridge University Press, Cambridge (2010).

[3] Boulos, M. N. K., Wheeler, S., Tavares, C. and Jones, R., "How smartphones are changing the face of mobile and participatory healthcare: an overview, with example from eCAALYX," Biomed. Eng. OnLine 10, 24-37 (2011).

[4] Hicks, J., "Smartphone app diagnoses malaria from drop of blood," Forbes, 3 January 2012, < http://www.forbes.com/sites/jenniferhicks/2012/01/03/smartphone-app-diagnoses-malaria-from-drop-ofblood/> (26 April 2012).

[5] US Department of Homeland Security Official Web Page, "Cell-All: Super smartphones sniff out suspicious substances," < www.dhs.gov/cell-all-super-smartphones-sniff-out-suspicious-substances> (29 August 2012).

[6] Wang, S. X. and Zhou, X. J. (to BWT Property Inc., NJ), "Spectroscopic sensor on mobile phone," US Patent $7,420,663$ (2008).

[7] Zhou, W. (to Shanghai Huaqin Telecom Technology), "Mobile phone with alcohol detection function," Chinese Patent 201,937,701(2011).

[8] Chae, H. S. (to SF Technology Co. Ltd, South Korea), "Smartphone-integrated environmental radioactivity measurement apparatus," Korean Patent 1,065,671 (2011).

[9] Chang, B.-Y., "Smartphone-based chemistry instrumentation: Digitization of colorimetric measurements," Bull. Korean Chem. Soc. 33, 549-552 (2012)..

[10]López-Ruiz, N., Martínez-Olmos, A., Pérez de Vargas-Sansalvador, I. M., Fernández-Ramos, M. D., Carvajal, M. A., Capitán-Vallvey, L. F. and Palma, A. J., "Determination of $\mathrm{O}_{2}$ using colour sensing from image processing with mobile devices," Sensors Actuators B: Chemical 171-172, 938-945 (2012).

[11] Marimuthu, M., Kandasamy, K., Ahn, C. G., Sung, G. Y., Kim, M.-G. and Kim, S., "CMOS image sensor for detection of interferon gamma protein interaction as a point-of-care approach," Anal. Bioanal. Chem. 401, 1641-1649 (2011).

[12]Morkoç, H., [Handbook of Nitride Semiconductors and Devices, Vol. 3: GaN-based Optical and Electronic Devices], Wiley-VCH, Weinheim (2009).

[13]López-Gejo, J., Arranz, A., Navarro, A., Palacio, C., Muñoz, E. and Orellana, G., "Microsensors Based on GaN Semiconductors Covalently Functionalized With Luminescent Ru(II) Complexes," J. Am. Chem. Soc. 132, 1746-1747 (2010).

[14] Orellana, G. and García-Fresnadillo, D., "Environmental and industrial optosensing with tailored luminescent Ru(II) polypyridyl complexes," in [Optical Sensors: Industrial, Environmental and Diagnostic Applications], Narayanaswamy, R. and Wolfbeis, O. S. Eds., Springer Series in Chemical Sensors and Biosensors, Vol. 1, Springer, Berlin-Heidelberg, 309-357 (2004).

[15] Orellana, G., Moreno-Bondi, M. C., García-Fresnadillo, D. and Marazuela, M. D., "The interplay of indicator, support and analyte in optical sensor layers", in [Frontiers in Chemical Sensors: Novel Principles and Techniques], Orellana, G. and Moreno-Bondi, M.C. Eds., Springer Series in Chemical Sensors and Biosensors, Vol. 3, Springer, Berlin-Heidelberg, Alemania; pp 189-225 (2005).

[16]López-Gejo, J., Arranz, A., Navarro, A., Palacio, C., Muñoz, E. and Orellana, G., "Direct Grafting of LongLived Luminescent Indicator Dyes to GaN Light-Emitting Diodes for Chemical Microsensor Development," ACS Appl. Mater. Interf. 3, 3846-3854 (2011).

[17] Chu, C., Cheng, C., Liu, W., Chu, J., Fan, F., Cheng, H. and Tran, C., "High Brightness GaN Vertical LED on Metal Alloy for General Lighting Applications," Proc. IEEE 98, 1197-1207 (2010).

[18] Arranz, A., Palacio, C., García-Fresnadillo, D., Orellana, G., Navarro A. and Muñoz, E., "Influence of Surface Hydroxylation on 3-Aminopropyltriethoxysilane Growth Mode during Chemical Functionalization of GaN Surfaces: An Angle-Resolved X-ray Photoelectron Spectroscopy Study," Langmuir 24, 8667-8671 (2008).

[19]Zanarini, S., Ciana, L. D., Marcaccio, M., Marzocchi, E., Paolucci, F. and Prodi, L. "Electrochemistry and Electrochemiluminescence of $[\mathrm{Ru}(\mathrm{II}) \text {-tris(bathophenanthroline-disulfonate) }]^{4-}$ in Aprotic Conditions and Aqueous Buffers," J. Phys. Chem. B 112, 10188-10193 (2008). 
[20] Smestada, G. P., Spiekermannb, S., Kowalik, J., Grantd, C. D., Schwartzbergd, A. M., Zhang, J., Tolbert, L. M. and Moons, E., "A technique to compare polythiophene solid-state dye sensitized $\mathrm{TiO} 2$ solar cells to liquid junction devices," Solar Energy Mater. \& Solar Cells 76, 85-105 (2003).

[21] Moses, P. and Van de Walle, C., "Band bowing and band alignment in InGaN alloys," Appl. Phys. Letters 96, $021908(2010)$.

[22] Nemanich, R. J., Benjamin, M. C., Bozeman, S. P., Bremser, M. D., King, S. W., Ward, B. L., Davis, R. F., Chen, B., Zhang, Z. and Bernholc, J., "(Negative) electron affinity of AlN and AlGaN alloys," MRS Proc. 395, 777, 1-12 (1995) (doi:10.1557/PROC-395-777).

[23] Carlson, B., Leschkies, K., Aydil, E. S. and Zhu, X.-Y., "Valence band alignment at Cadmium Selenide Quantum Dot and Zinc Oxide (10I0) Interfaces," J. Phys. Chem. C 112, 8419-8423 (2008).

[24]Zhai, H.-J. and Wang L.-S., "Probing the electronic structure and band gap evolution of Titanium Oxide clusters $\left(\mathrm{TiO}_{2}\right)_{\mathrm{n}}(\mathrm{n}=1-10)$ using photoelectron spectroscopy," J. Am. Chem. Soc. 129, 3022-3026 (2007).

[25] Margalith, T. and Buchinsky, O., "Indium tin oxide contracts to gallium nitride optoelectronic devices," Appl. Phys. Letters, 74, 3930-3934 (1999).

[26]Lin, S. C., Kuo, C.-T., Liu, X., Liang, L.-Y., Cheng, C.-H., Lin, C.-H., Tang, S.-J., Chang, L.-Y., Chen, C.-H. and Gwo, S., "Experimental Determination of Electron Affinities for InN and GaN Polar Surfaces," Appl. Phys. Express 5, 031003 (2012). 